\title{
The use of goggles for testing hemispheric asymmetry
}

\author{
JAMES B. FRANCKS, STEVEN M. SMITH, and THOMAS B. WARD \\ Texas A\&M University, College Station, Texas
}

\begin{abstract}
A technique for restricting visual stimuli to a single visual field was validated in the present study. Vertical strips of opaque tape were applied to close-fitting goggles, obscuring one visual field on each lens. Forty-seven right-handed participants fitted with such goggles were found to be faster and more accurate in processing verbal stimuli presented to the right visual field than to the left. For a spatial task, accuracy was better and reaction times were shorter when stimuli were presented to the left visual field. Results indicate that the taped goggles were successful enough in restricting input to a single visual field to replicate typical laterality effects.
\end{abstract}

Techniques for studying hemispheric asymmetries have varied considerably. An early experimental method was Mishkin and Forgays's (1952) technique of tachistoscopically presenting a stimulus to one visual field or the other. In recent years, a variety of other procedures have been developed, such as auditory dichotic presentations (Kimura, 1961), electroencephalograms or EEGs (Galin \& Ornstein, 1972), measures of blood flow in the cerebral hemispheres (Lassen \& Ingvar, 1972), and visually restricting contact lenses (Zaidel, 1975). All of these alternatives to the tachistoscope are either expensive, timeconsuming, or procedurally complicated.

The experimenter desiring to present visual stimuli unilaterally has had only two techniques available for research with "intact" humans. These techniques require precisely positioned lateral presentations using a tachistoscope or partially covered contact lenses. The tachistoscopic presentations must be located a certain exact number of degrees off center. In addition, either the presentations must be very brief in order to preclude eye movements, or the experimenter must constantly monitor eye position. Because partially covered contact lenses block one visual field, the problems of eye movements and precise stimulus placement are less critical. The lenses, however, may be expensive and not readily available.

The present study validated a technique for restricting an image to one visual field which requires only a pair of snugly fitting goggles and some opaque tape. Once the goggles are fitted to the participant's face, vertical strips of tape are used to cover half of each lens, including approximately one half of each pupil. In this way it is possible to substantially restrict stimuli from one visual field such that visual input can be channeled primarily to one hemisphere.

This study was part of a master's thesis by the first author. Requests for reprints should be sent to Steven M. Smith, Department of Psychology, Texas A\&M University, College Station, TX 77843-4235.
Taped goggles were used in the present study to replicate established findings for recognition of tachistoscopically presented verbal material (White, 1969), and to replicate an experiment conducted by Kimura (1961) using a dot-localization task. For right-handed individuals, the left hemisphere is supposedly better at processing verbal material and the right hemisphere is supposedly better at processing spatial material. If the goggles are successful in limiting presentations to one visual field, such laterality effects should be observed in the present study.

\section{METHOD}

\section{Participants}

Participants were 47 right-handed undergraduate students $(27$ males and 20 females) who participated in order to fulfill a course requirement in an introductory psychology course at Texas A\&M University.

\section{Apparatus and Materials}

Stimuli were presented via a Lafayette Instrument Constant Illumination Electronic Tachistoscope, Model 42011. It was equipped with two Kodak slide projectors. Brief visual presentations were used to avoid ceiling effects on accuracy measures of word recognition. A timer, which recorded response times, was activated by a photosensitive cell located in front of the slide projector which presented the stimuli. The timer was stopped by a voice-response microphone (Lafayette Voice Response Time Control, Model 63040) worn around the participant's neck. Two pairs of Speedo swimming goggles (Model 6501) were used, and black tape was vertically arranged to cover half of each lens.

Verbal stimuli were 52 high-frequency four-letter nouns typed in uppercase letters with an IBM Selectric typewriter. The words were printed vertically and centered on slides. Spatial stimuli consisted of 52 different slides with a single dot in one of 52 possible positions in a circular array of dots. A response card containing all 52 dot positions along with individual identifying numbers was prepared on a white $7.6 \times 12.7$ $\mathrm{cm}$ index card.

\section{Procedure}

Each participant was seated in a straight-back chair and was instructed to rest the back of his/her head on two vertical rods (spaced 4 in. apart) mounted on the back of the chair. Participants were thus restricted from moving their heads or eyes to positions where stimuli could be viewed in both visual fields. Each participant was fitted with two separate pairs of goggles. Black tape was placed vertically over half of each lens so that only half of the participant's pupil was visible. Pilot observations indicate that this procedure was effective in blocking all but $0.5^{\circ}$ or 
less of the obstructed visual field. One pair of goggles had tape over the participant's right visual field, and the other had tape over his/her left visual field. Each participant was instructed to fixate on the center dot before each trial. The stimuli were projected on a white wall approximately $3 \mathrm{ft}$ directly in front of the participant for an exposure interval of $1 / 150$ of $1 \mathrm{sec}$.

The verbal and spatial tasks were presented in blocks of eight trials. The type of task and visual field exposed were randomized to minimize any order or practice effects. Four practice trials were administered. After each exposure during the verbal task, the participant was to say the stimulus word aloud. For the spatial task, the participant was to say the number associated with the dot that was exposed. During the spatial task, the participant held the matrix card in a comfortable position and was reminded not to move his/her head from the vertical rods. Reaction times were determined as the duration of time from the initial exposure until the participant made a verbal response. Accuracy was determined by the number of correct responses based on a maximum score of $\mathbf{5 2}$ for each task.

\section{RESULTS}

Two separate analyses of variance were computed, one for response accuracy and one for reaction time, using visual field $(2) \times$ stimulus type $(2) \times$ stimulus order $(2)$ $X$ visual field order (2) as independent variables. Visual field and stimulus type were repeated measures (each subject received all possible combinations), and stimulus order and visual field order were grouping factors. Visual field was either left or right, stimulus type was either spatial or verbal, stimulus order was either spatial first or verbal first, and visual field order was either left first or right first.

As shown in Table 1, performance on the verbal task was better when stimuli were presented to the right visual field (RVF) than to the left visual field (LVF). For the spatial task, performance was better for the LVF than for the RVF. These effects were found on both measures of accuracy and reaction time. The stimulus type $\times$ visual

Table 1

Mean Number of Correct Responses and Reaction Time (RT) for Left and Right Visual Fields on Verbal and Spatial Tasks

\begin{tabular}{lrr} 
& \multicolumn{2}{c}{ Visual Field } \\
\cline { 2 - 3 } Task & \multicolumn{1}{c}{ Left } & \multicolumn{1}{c}{ Right } \\
\hline Verbal & 17.75 & \\
Accuracy & 978.81 & 19.04 \\
RT (msec) & & 902.62 \\
Spatial & 6.92 & \\
Accuracy & 1893.43 & 5.70 \\
RT (msec) & 2086.38 \\
\hline
\end{tabular}

Note-The maximum possible accuracy score was 52 for both verbal and spatial tasks. field interaction was significant both for accuracy measures $[F(1,39)=38.61, p<.001]$ and for reaction times $[F(1,39)=14.90, p<.001]$. Newman-Keuls tests $(r=$ $2, \mathrm{p}<.05$, critical difference $=.56)$ confirmed that verbal stimuli were more accurately named when presented to the RVF than to the LVF (mean difference $=1.29$ ), and that performance on spatial stimuli was more accurate for the LVF than for the RVF (mean difference $=1.22$ ). For reaction-time measures $(\mathrm{r}=2, \mathrm{p}<.05$, critical difference $=97.84$ ), the advantage of the LVF over the RVF on the spatial task was significant (mean difference $=192.95$ ), although the RVF-LVF difference on the verbal task did not quite reach significance (mean difference $=76.19$ ).

\section{DISCUSSION}

The overall results of this experiment were similar to findings reviewed by White (1969) for verbal tasks and Kimura's (1961) findings using the spatial task. As predicted, the RVF was found to be superior to the LVF for verbal stimuli in terms of accuracy and reaction time, whereas the LVF was found to be superior to the RVF for spatial stimuli. An alternative hypothesis, that one visual field is always more accurate, is clearly disproven, because each visual field was better for processing one stimulus type or the other. The results suggest that the taped goggles were successful enough in restricting input to a single visual field to replicate standard laterality effects. Thus, taped goggles provide an alternative to using visually restricting contact lenses, monitoring blood flow in the cerebral hemispheres, and making EEG recordings. The simplicity and economy involved should allow more investigators to study hemispheric asymmetries in the processing of information. Although the taping procedure is inexact $\left(\leq .5^{\circ}\right)$, accuracy can be enhanced by enlisting the cooperation of the participant. The participant's head should be kept as stationary as possible. With one eye closed, the participant should look directly at a fixation point and indicate to the experimenter when the edge of the tape which is being applied just reaches the fixation point. This procedure should be repeated separately for each eye.

\section{REFERENCES}

GALIN, D., \& ORNSTEIN, R. (1972). Lateral specialization of cognitive mode: An EEG study. Psychophysiology, 9, 412-418.

KimuRA, D. (1961). Some effects of temporal lobe damage on auditory perception. Canadian Journal of Psychology, 15, 156-165.

LASSEN, N. A., \& INGVAR, D. H. (1972). Radioisotopic assessment of regional cerebral blood flows. In A. Donath \& A. N. Serafini (Eds.), Progress in Nuclear Medicine, (pp. 376-409). Baltimore: University Park Press.

MishKIN, M., \& Forgays, D. B. (1952). Word recognition as a function of retinal locus. Journal of Experimental Psychology, 43, 43-48.

White, M. J. (1969). Laterality differences in perception: A review. Psychological Bulletin, 72, 387-405.

ZAIDEL, E. (1975). A technique for presenting lateralized visual input with prolonged exposure. Vision Research, 15, 283-289.

(Manuscript received for publication August 15, 1985.) 